\title{
Anfibios y reptiles del valle del Mezquital, Hidalgo, México
}

\section{Amphibians and reptiles from the Valle del Mezquital, Hidalgo, Mexico}

\author{
Leonardo Fernández-Badillo* e Irene Goyenechea-Mayer Goyenechea \\ Centro de Investigaciones Biológicas, Universidad Autónoma del Estado de Hidalgo. Ciudad Universitaria, Carr. Pachuca Tulancingo Km. 4.5, \\ 42184 Pachuca, Hidalgo, México. \\ *Correspondencia: cyrtopsis@hotmail.com
}

\begin{abstract}
Resumen. El valle del Mezquital, Hidalgo, México, es un área de gran riqueza biológica; sin embargo, se desconoce mucho sobre su herpetofauna, por lo que se realizó una lista de anfibios y reptiles de 3 zonas del valle. Se analizó su distribución por tipo de vegetación, se registraron los microhábitats utilizados, así como la abundancia relativa. Se llevaron a cabo 12 salidas mensuales durante 1 año, con 108 días de muestreo. Se realizaron recorridos sobre transectos, en 9 tipos de vegetación. La herpetofauna del área de estudio está integrada por 37 especies (7 anfibios y 30 reptiles), de las cuales 8 se registran por primera vez para el valle del Mezquital. La mayoría de las especies se presentaron en las zonas de cultivo (26), 14 especies en los de la zona templada y 13 en los de la zona de riego. La herpetofauna del valle del Mezquital utiliza un total de 27 tipos de microhábitats, siendo "bajo roca" donde se registró el mayor número de especies (22). La mayoría de las especies tanto de anfibios como de reptiles fueron consideradas raras.
\end{abstract}

Palabras clave: diversidad, herpetozoos, primeros registros.

\begin{abstract}
Valle del Mezquital Hidalgo, Mexico is known for its high biological richness, but little is known about its herpetofauna. A study to list the amphibians and reptiles of 3 different zones at the Valle del Mezquital was carried out. We analyzed their distribution in different types of vegetation, their microhabitat and relative abundance in 12 monthly trips during 1 year for a total of 108 days. We sampled transects over 9 types of vegetation. Thirty species have been reported for this area (7 amphibians, 30 reptiles), 8 reported for the first time from Valle del Mezquital. Most of the species were present within crops (26), 14 within the temperate zone and 13 within the irrigation zone. The herpetofauna at Valle del Mezquital uses 27 different microhabitat, being "under rock" the most used by 22 species. Most of the species, both amphibians and reptiles were considered rare.
\end{abstract}

Key words: diversity, herptozoos, first records.

\section{Introducción}

El valle del Mezquital, Hidalgo, abarca 27 municipios (INEGI, 1998) lo que representa el $33.7 \%$ de la superficie del estado de Hidalgo (Arroyo, 2001); constituye un área de gran importancia ecológica, económica, social, histórica y cultural que se caracteriza por sus diversas condiciones ambientales lo que ocasiona que se presenten gradientes ecológicos que favorecen la presencia de diversos hábitats (López, 2001). Las variantes altitudinales, fisiográficas, de sustrato y suelo influyen de manera decisiva en el establecimiento de variadas formas de vida y en el desarrollo de una gran riqueza específica, lo que genera mosaicos de vegetación con un amplio potencial de

Recibido: 17 agosto 2009; aceptado: 12 enero 2010 recursos para la zona (López, 2001).

El valle del Mezquital junto con el desierto chihuahuense, la zona de Baja California, y el valle de Tehuacán-Cuicatlán, Puebla/Oaxaca conforman las 4 grandes regiones áridas y semiáridas del país (Shreve, 1942). Las zonas áridas comprenden alrededor del $60 \%$ del total del territorio nacional, y aunque menos ricas que las regiones tropicales, son de gran valor, ya que presentan una enorme diversidad de especies vegetales. Con excepción del desierto sonorense, han sido poco estudiadas y se encuentran bajo amenaza por la acelerada perturbación a causa de las actividades humanas, como la sobreexplotación de la cobertura vegetal, la erosión del suelo y el sobrepastoreo (Rzedowski, 1996), y aun cuando el estudio de los anfibios y reptiles del país se ha visto enriquecido en los últimos años, hay una gran 
carencia de información para ciertas zonas en cuanto al número de especies, lo cual se ve reflejado en la falta de inventarios actualizados. Este tipo de estudios son los que dan la pauta para llevar a cabo medidas enfocadas a la protección y conservación ante la rápida destrucción de los hábitats naturales, situación que resulta alarmante si se considera que es imposible proteger o preservar especies si se desconoce su distribución, y es imposible emprender estrategias de conservación en aquellos hábitats de los que aún no se conoce la diversidad de especies, de ahí la importancia de ampliar el conocimiento en zonas poco estudiadas y con importancia ecológica como lo es el valle del Mezquital.

En el presente estudio se realizó una lista de las especies de anfibios y reptiles en 3 zonas diferentes del valle del Mezquital, Hidalgo. Se analizó la riqueza y la distribución de las especies por tipo de vegetación, se identificaron los tipos de microhábitat que utilizan cada una de las especies y se estimó la abundancia relativa de las especies que habitan en el área de estudio.

\section{Materiales y métodos}

El estudio se llevó a abo en el periodo comprendido entre febrero de 2005 y enero de 2006, en el alto Mezquital (López, 2001), específicamente en el municipio de Ixmiquilpan, Hidalgo, $\left(20^{\circ} 41^{\prime}\right.$ y $20^{\circ} 31^{\prime} \mathrm{N}$ y $99^{\circ} 15^{\prime}$ y $99^{\circ} 13^{\prime} \mathrm{O}$; Fig. 1). La zona registra un gradiente ambiental que propicia la presencia de 2 tipos de clima. En las zonas de mayor altitud, entre los 2000 y 2800 m, el clima es templado húmedo con lluvias en verano, en tanto que entre los 1600 y los $2000 \mathrm{~m}$ el clima es semiseco estepario (García, 1973).

El área de estudio se dividió en 3 zonas (zona de riego de 1600 a 1 700m; zona árida

de 1750 a $2000 \mathrm{~m}$, y zona templada de 2400 a $2800 \mathrm{~m}$ ). Para conocer la riqueza de las especies en los 9 tipo de vegetación (Fig. 1), se realizaron salidas mensuales de 9 días cada una durante un año, con un esfuerzo de captura de 7 hrs/hombre $\times$ día, tomando datos de presencia y ausencia de las especies, incluyendo organismos recolectados y

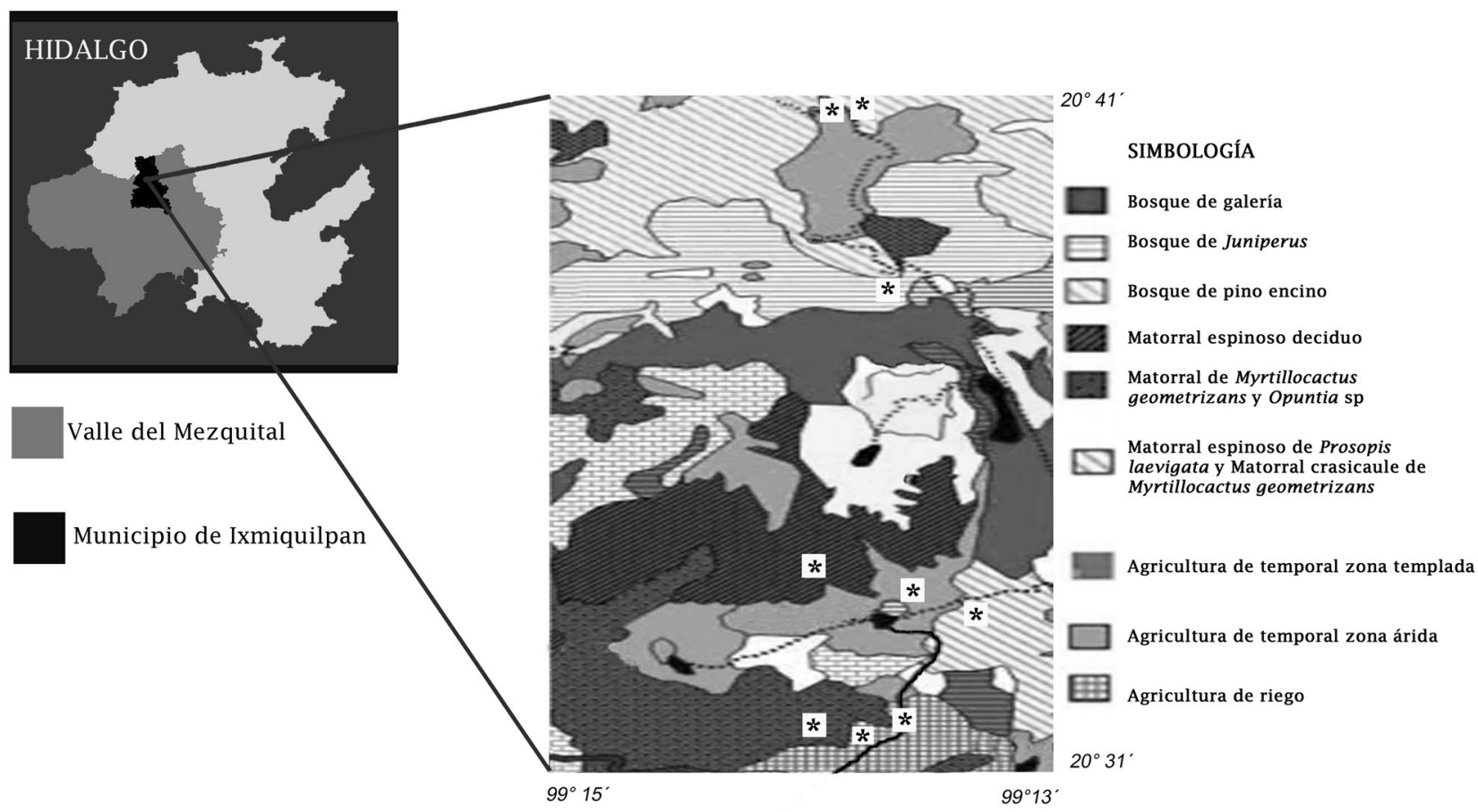

Figura 1. Mapa de tipos de vegetación del municipio de Ixmiquilpan, Hidalgo. Los asteriscos indican las zonas donde se llevaron a cabo los muestreos del presente estudio. 
observados. Para ello, se estableció un transecto en línea (Camarillo, 1981) con una extensión de $1000 \mathrm{~m} \times 10 \mathrm{~m}$, uno por cada tipo de vegetación.

La captura de los organismos se llevo a cabo con permiso de colector científico FAUT-0052, utilizándose las técnicas descritas por Casas et al. (1991) Cuando no fue posible identificarlos en campo, los ejemlares fueron sacrificados (Martínez y Muñoz, 1998) y posteriormente se depositaron en la colección herpetológica del Centro de Investigaciones Biológicas de la Universidad Autónoma del Estado de Hidalgo.

Se obtuvo una curva de acumulación de especies empleando los estimadores Jack de primer orden y Bootstrap (Colwell y Coddington 1994), graficando además las especies que aparecen sólo una vez (singletons), así como las que aparecen 2 veces (doubletons). Estos estimadores resultaron los más adecuados para este estudio,ya que permitieron interpretar de la mejor manera los datos (Moreno, 2001).

La abundancia relativa de las poblaciones se obtuvo a partir del total de registros (organismos observados, recolectados y no recolectados), siguiendo los mismos criterios empleados por Duellman (1965), Muñoz (1988), Mendoza (1990), Martínez y Muñoz (1998) y Vargas (1998) donde una especie se considera rara (R) cuando se registran uno o 2 ejemplares; moderadamente abundante (M) si se observan de 3 a 5 ejemplares, y abundante (A) cuando se encuentran más de 5 ejemplares.

\section{Resultados}

En la porción estudiada del valle del Mezquital, Hidalgo se encontró un total de 37 especies (Cuadro 1), las cuales constituyen 5 familias, 6 géneros y 7 especies de anfibios, así como 8 familias, 24 géneros y 30 especies de reptiles. Se registran por primera vez para el valle del Mezquital los reptiles Sceloporus scalaris, Ficimia hardyi, Pantherophis emoryi, Rhadinaea hesperia, Salvadora grahamiae, Storeria hidalgoensis, Thamnophis melanogaster y Crotalus aquilus. Con el total de las especies registradas a lo largo del estudio se obtuvo una curva de acumulación de especies alcanzando el $80 \%$ de las especies previstas por los estimadores (Fig. 2).

En lo referente a la riqueza de especies por tipo de vegetación (Cuadro 1), fue en los cultivos de la zona templada (i) donde se registró la mayor cantidad de especies, un total de 14, seguidos por los cultivos de la zona de riego (c) con 13; el bosque de encino (g) con 10; el matorral espinoso deciduo (d) con 7; el matorral espinoso de Prosopis laevigata y Mirtyllocactus geometrizans (f) con 6 , y por último, en los tipos de vegetación restantes se registraron 4 especies.

A lo largo de las distintas áreas marcadas en la zona de estudio se encontraron 27 microhábitats, 9 de los cuales son utilizados por especies de anfibios. El más utilizado es "bajo roca", donde se observaron 3 especies (Anaxyrus punctatus, Chiropterotriton sp. y Craugastor sp.). También fue "bajo roca" el más utilizado por los reptiles, registrándose 19 especies en total, integrado por 8 especies de lacertilios y 11 de serpientes (Aspidoscelis gularis, Barisia imbricata, Plestiodon lynxe, Sceloporus grammicus, S. parvus, S. scalaris, S. spinosus, S. torquatus, Conopsis lineata, Crotalus aquilus, Ficimia hardyi, Geophis semianulatus, Hypsiglena jani, Rena dulcis, Coluber schotti, Pantherophis emoryi, Pituophis deppei, Salvadora grahamiae y Storeria hidalgoensis). En segundo lugar se utilizó el microhábitat "sobre suelo", donde se registraron 12 especies, 7 de lagartijas y 5 de serpientes (Aspidoscelis gularis, Phrynosoma orbiculare, Sceloporus grammicus, S. parvus, S. scalaris, S. spinosus, S. torquatus, Rhadinaea. hesperia, Salvadora grahamiae, Trimorphodon tau, Crotalus atrox y Tropidodipsas sartorii). Se encontraron 3 microhábitats en los que sólo se registró 1 especie, y finalmente 4 también con un solo registro, pero de especie distinta en cada uno. El microhábitat más abundante en cuanto a número de individuos fue "sobre suelo" con 180 organismos y, por el contrario, 7 microhábitats distintos sólo presentaron un individuo.

Del total de las especies encontradas en campo se registraron 21 especies en la categoría de raras, lo que representa el $56.7 \%$ de la herpetofauna total. El 16.2\% son poblaciones moderadamente abundantes, donde se incluyen 6 especies. Finalmente, el $27.02 \%$ está integrado por 10 especies que corresponden a la herpetofauna cuyas poblaciones se presentan en forma abundante (Cuadro 1).

\section{Discusión}

De las 37 especies encontradas a lo largo del estudio, 7 corresponden a anfibios, de éstos, 5 son del orden Anura; Caudata estuvo representado únicamente por 2 especies. Del total de la herpetofauna, el grupo más diverso fue Serpentes con 24 especies, 15 de las cuales son de Colubridae, la familia mejor representada; fue seguido por Sauria con 11 especies, de las cuales 7 pertenecen a la familia Phrynosomatidae.

El número de especies registrado muestra la elevada riqueza herpetofaunística de la zona, lo cual resulta claro al comparar estos datos con los obtenidos por otros autores para las zonas áridas del estado de Hidalgo. En 2 años consecutivos, Martín del Campo $(1936,1937)$ registró 14 y 17 especies respectivamente; Mendoza (1990) encontró 
Cuadro 1. Lista de especies, tipo de vegetación, tipo de microhábitat y abundancia relativa de la herpetofauna registrada en 3 zonas del valle del Mezquital, Hidalgo, México

\begin{tabular}{|c|c|c|c|}
\hline Especies & Vegetación & Microhábitat & Abundancia \\
\hline Anaxyrus punctatus & $\mathrm{d}$ & 2 & Rara \\
\hline Hyla arenicolor & $\mathrm{i}$ & 1 & Rara \\
\hline Hyla eximia & I & 27 & Rara \\
\hline Craugastor sp. & $\mathrm{g}$ & 2 & Rara \\
\hline Lithobates spectabilis & $\mathrm{a}, \mathrm{b}, \mathrm{c}, \mathrm{i}$ & $16,17,20,21,23$ & Abundante \\
\hline Pseudoeurycea cephalica & $\mathrm{i}$ & 24 & Rara \\
\hline Chiropterotriton sp. & g & 2 & Rara \\
\hline Barisia imbricata & $\mathrm{g}, \mathrm{i}$ & $2,4,6,7,27$ & Abundante \\
\hline Gerrhonotus infernalis & $\mathrm{d}$ & 12 & Rara \\
\hline Phrynosoma orbiculare & $\mathrm{i}$ & 9,16 & Rara \\
\hline Sceloporus grammicus & g, i & $1,2,4,5,6,8,9,10,15,16,26$ & Abundante \\
\hline Sceloporus minor & $\mathrm{g}$ & 1 & Rara \\
\hline Sceloporus parvus & $\mathrm{c}, \mathrm{d}, \mathrm{f}, \mathrm{h}$ & $1,2,6,12,13,16$ & Abundante \\
\hline Sceloporus scalaris & $\mathrm{i}$ & $1,2,7,13,16,23,24$ & Abundante \\
\hline Sceloporus spinosus & $a, b, c, d, e, f$ & $1,2,11,13,16,25$ & Abundante \\
\hline Sceloporus torquatus & $\mathrm{g}, \mathrm{i}$ & $1,2,3,4,6,10,15,16,22,27$ & Abundante \\
\hline Plestiodon lynxe & $\mathrm{i}$ & 2 & Abundante \\
\hline Aspidoscelis gularis & $a, b, c, d, e, f$ & $2,11,12,13,14,16$ & Abundante \\
\hline Conopsis lineata & $\mathrm{g}$ & 2 & Rara \\
\hline Diadophis punctatus & $i$ & 7 & Rara \\
\hline Drymarchon melanurus & $\mathrm{c}$ & 18 & Rara \\
\hline Ficimia hardyi & $\mathrm{d}$ & 2 & Rara \\
\hline Geophis semiannulatus & $\mathrm{g}, \mathrm{i}$ & 2 & Rara \\
\hline Hypsiglena jani & $\mathrm{d}, \mathrm{f}$ & 2 & Moderada \\
\hline Coluber schotti & $c, f, g$ & $2,6,11,19$ & Moderada \\
\hline Pantherophis emoryi & $\mathrm{b}, \mathrm{c}$ & 1,2 & Rara \\
\hline Pituophis deppei & $e, f$ & 2,11 & Rara \\
\hline Rhadinaea hesperia & $\mathrm{g}$ & 16 & Rara \\
\hline Salvadora grahamiae & $\mathrm{c}, \mathrm{e}, \mathrm{g}$ & $2,16,19$ & Moderada \\
\hline Storeria hidalgoensis & $\mathrm{i}^{\circ}$ & 2,22 & Moderada \\
\hline Thamnophis melanogaster & a & 20 & Rara \\
\hline Trimorphodon tau & $\mathrm{c}$ & 16 & Rara \\
\hline Tropidodipsas sartorii & $\mathrm{c}$ & 16 & Moderada \\
\hline Rena dulcis & $\mathrm{c}$ & 2 & Rara \\
\hline Crotalus aquilus & $\mathrm{g}, \mathrm{i}$ & $1,2,7,19$ & Moderada \\
\hline Crotalus atrox & $\mathrm{c}$ & 16 & Rara \\
\hline Kinosternon hirtipes & $\mathrm{c}$ & 4 & Abundante \\
\hline
\end{tabular}

Vegetación: a, bosque de galería; b, matorral crasicaule de Myrtillocactus geometrizans y Opuntia sp.; c, agricultura de riego; d, matorral espinoso deciduo; e, agricultura de temporal, zona árida; f, matorral espinoso de Prosopis laevigata con matorral crasicaule de M. geometrizans; g, bosque de encino; h,bosque de Juniperus; i, agricultura de temporal, zona templada. Microhábitats: 1, sobre roca; 2 , bajo roca; 3 , entre rocas; 4 , sobre troncos; 5 , bajo troncos; 6 , sobre maguey seco; 7 , bajo maguey seco; 8 , sobre maguey; 9 , bajo maguey; 10, entre magueyes; 11, entre lechuguillas; 12 , entre junquillos; 13, bajo arbustos; 14, entre nopales; 15, sobre árbol; 16, sobre suelo; 17 , bajo cactus; 18 , entre carrizos; 19, bajo lamina; 20, dentro del agua; 21 , orillas del río; 22, bajo pasto; 23, entre pasto; 24 , sobre pasto; 25 , entre huapillas; 26 , sobre zacate; 27 , bajo zacate.

31 en el transecto Zacualtipán- Zoquizoquipan-San Juan Meztitlán; Camarillo (1993) menciona 39 para toda la zona xerófila del estado de Hidalgo; Gelover et al. (2000), 13 para la zona de Meztitlán; Huitzil (2007), 17 para la región norte de Zimapán, y Vite (2008) registró 24 en la Reserva de la Biosfera Barranca de Meztitlán (Cuadro 2).
Se registraron 8 especies por primera vez para el valle del Mezquital, cuya presencia muestra lo necesario de estos estudios y de lo mucho que falta por conocer acerca de la diversidad y distribución de los anfibios y reptiles, así como la gran riqueza biológica que habita en los ambientes semiáridos que tan escasamente han sido 


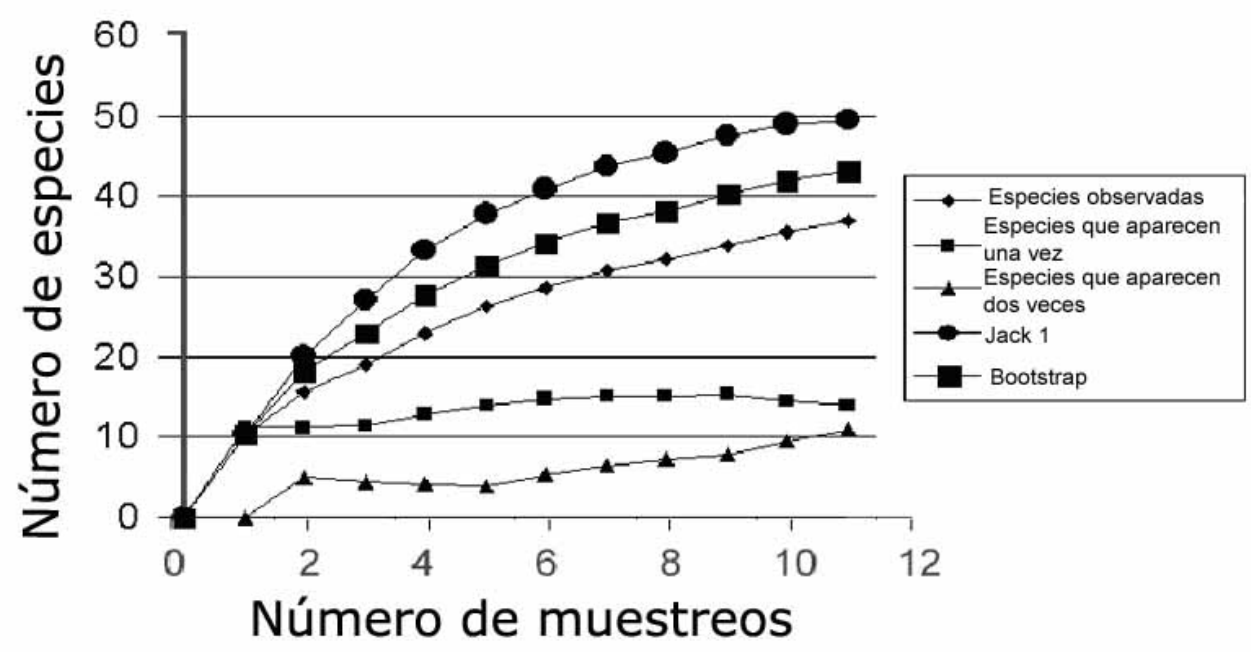

Figura 2. Curva de acumulación de especies a lo largo de los muestreos en el valle del Mezquital, la cual alcanzó el $80 \%$ de las especies previstas por los estimadores.

estudiados en el estado de Hidalgo.

La curva de acumulación de especies (Fig. 2) alcanzó el $80 \%$ de las especies previstas por los estimadores, los registros sobre los singletons y doubletons casi se cruzan, lo que permite considerar que el área de estudio fue muestreada adecuadamente; sin embargo, es recomendable durante la temporada de lluvias realizar un par de muestreos más a fin de localizar aquellas especies que durante el año de muestreo no fue posible registrar por la escasez de lluvias.

La mayor cantidad de especies (14) se registró en los cultivos de la zona templada. Este tipo de vegetación presenta un gran número de microhábitats, producto de la alteración causada por el hombre, así como cuerpos de agua artificiales que atraen a anfibios y a reptiles; asimismo, otro factor que atrae a diferentes especies de serpientes y lagartijas es la disponibilidad de alimento, y dicha zona alberga un alto número roedores e insectos, lo que concuerda con lo observado por González (1999), quien menciona que la abundancia de granos y otras plantas en las zonas de cultivo favorece la proliferación de roedores, lo que atrae a una variedad de serpientes que se alimentan tanto de éstos como de lagartijas e insectos.

Los cultivos de la zona de riego, con 13 especies, fue el segundo tipo de vegetación en cuanto a número de especies. En dichas áreas, los cuerpos de agua permanentes, los canales y la cercanía al río Tula, favorecen la presencia de reptiles como Drymarchon melanurus, Kinosternon hirtipes, Thamnophis melanogaster y Tropidodipsas sartorii, cuyos hábitos se encuentran ligados a los cuerpos de agua y a zonas de mucha de humedad. Por el contrario, las especies de anfibios pudieron verse restringidas, debido a que las tierras sólo se irrigan por las noches, estando secas durante el día; además, los canales de riego, carecen de vegetación y la corriente es rápida, lo que impide la formación de charcas, y las pocas que llegan a formarse a las orillas de los canales están ocupadas por Lithobates spectabilis. Aunado a ello, las aguas del río Tula así como las que corren por los pequeños arroyos y los canales de riego se encuentran altamente contaminadas, puesto que son aguas negras provenientes en gran parte de descargas municipales de la ciudad de México y áreas conurbadas (López, 2001), lo que afecta directamente a los anfibios, particularmente a las ranas, que son organismos altamente sensibles a los cambios ambientales a pequeña escala ((Duellman, 1999; Pineda et al., 2005).

En el bosque de encino se registraron 10 especies; 3 de ellas, Chiropterotriton sp., Craugastor sp. y la serpiente Conopsis lineata, presentan una distribución íntimamente ligada a las condiciones ambientales de este tipo de bosque. Las demás especies aquí registradas comparten las áreas de cultivo.

En cuarto lugar en cuanto a número de especies estuvo el matorral espinoso deciduo con 7 especies, siendo uno de los ambientes con menor número de microhábitats, lo que pudo haber limitado la presencia de anfibios y reptiles.

En el matorral espinoso de Prosopis laevigata con matorral crasicaule de Mirtyllocactus geometrizans se encontraron sólo 6 especies, todas de reptiles. Este tipo de ambiente está severamente afectado por la actividad humana, encontrándose sólo algunas especies, por ejemplo, Sceloporus parvus, S. spinosus, y Aspidoscelis gularis, las cuales parecieran no verse afectadas por la alteración humana (Mendoza, 1990; Vázquez-Díaz y Quintero-Díaz, 2005).

Por lo que respecta a los tipos de microhábitats utilizados 
Cuadro 2. Comparación de los registros de especies de anfibios y reptiles en el presente estudio y los de otros autores, en zonas áridas del estado de Hidalgo

\begin{tabular}{|c|c|c|c|c|c|c|c|c|c|c|c|c|c|c|c|}
\hline \multirow[t]{2}{*}{ Especies } & \multicolumn{7}{|c|}{ Fuentes } & \multirow[t]{2}{*}{ Especies } & \multicolumn{7}{|c|}{ Fuentes } \\
\hline & 1 & 2 & 3 & 4 & 5 & 6 & 7 & & 1 & 2 & 3 & 4 & 5 & 6 & 7 \\
\hline Anaxyrus punctatus & $\mathrm{x}$ & & & $\mathrm{x}$ & & & & Diadophis punctatus & $\mathrm{x}$ & & & & & & \\
\hline Incilius valliceps & & & & $\mathrm{x}$ & & & $\mathrm{x}$ & Drymarchon melanurus & $\mathrm{x}$ & $\mathrm{x}$ & $\mathrm{x}$ & $\mathrm{x}$ & $\mathrm{x}$ & & $\mathrm{x}$ \\
\hline Incilius occidentalis & & $\mathrm{x}$ & & & & $\mathrm{x}$ & & Ficimia hardyi* & $\mathrm{x}$ & & & & & & \\
\hline Rhinella marina & & & & & & & $\mathrm{x}$ & Ficimia streckeri & & & & $\mathrm{x}$ & & & \\
\hline Hyla arenicolor & $\mathrm{x}$ & $\mathrm{x}$ & & $\mathrm{x}$ & & $\mathrm{x}$ & & Ficimia variegata & & & $\mathrm{x}$ & & & & \\
\hline Hyla eximia & $\mathrm{x}$ & $\mathrm{x}$ & $\mathrm{x}$ & $\mathrm{x}$ & & & & Geophis latifrontalis & & & & & & $\mathrm{x}$ & \\
\hline Hyla myotimpanum & & & $\mathrm{x}$ & $\mathrm{x}$ & & & $\mathrm{x}$ & Geophis mutitorques & & & $\mathrm{x}$ & & & & \\
\hline Craugastor augusti & & & $\mathrm{x}$ & $\mathrm{x}$ & & & & Geophis semiannulatus & $\mathrm{x}$ & & & & & & $\mathrm{x}$ \\
\hline Eleutherodactylus longipes & & & & $\mathrm{x}$ & & $\mathrm{x}$ & & Hypsiglena jani & $\mathrm{x}$ & & & $\mathrm{x}$ & & & \\
\hline Eleutherodactylus verrucipes & & & & & & $\mathrm{x}$ & & Leptodeira septentrionalis & & & $\mathrm{x}$ & & $\mathrm{x}$ & & $\mathrm{x}$ \\
\hline Craugastor sp. & $\mathrm{x}$ & & & & & & & Coluber schotti & $\mathrm{x}$ & $\mathrm{x}$ & $\mathrm{x}$ & $\mathrm{x}$ & & $\mathrm{x}$ & \\
\hline Spea multiplicata & & $\mathrm{x}$ & $\mathrm{x}$ & $\mathrm{x}$ & & & $\mathrm{x}$ & Oxybelis aeneus & & & & & $\mathrm{x}$ & & \\
\hline Lithobates berlandieri & & & & $\mathrm{x}$ & & & $\mathrm{x}$ & Pantherophis emoryi* & $\mathrm{x}$ & & & & & & \\
\hline Lithobates montezumae & & $\mathrm{x}$ & & $\mathrm{x}$ & & & & Pituophis deppei & $\mathrm{x}$ & $\mathrm{x}$ & $\mathrm{x}$ & & & $\mathrm{x}$ & \\
\hline Lithobates spectabilis & $\mathrm{x}$ & $\mathrm{x}$ & $\mathrm{x}$ & & & & & Rhadinaea gaigae & & & $\mathrm{x}$ & & & & \\
\hline Ambystoma tigrinum & & $\mathrm{x}$ & & & & & & Rhadinaea hesperia* & $\mathrm{x}$ & & & & & & \\
\hline Ambystoma velasci & & & & $\mathrm{x}$ & & & & Salvadora grahamiae* & $\mathrm{x}$ & & & & & & \\
\hline Pseudoeurycea bellii & & $\mathrm{x}$ & & $\mathrm{x}$ & & & & Senticolis triaspis & & & & $\mathrm{x}$ & $\mathrm{x}$ & & $\mathrm{x}$ \\
\hline Pseudoeurycea cephalica & $\mathrm{x}$ & & $\mathrm{x}$ & & & $\mathrm{x}$ & & Storeira dekayi & & & $\mathrm{x}$ & & & & \\
\hline Chiropterotriton dimidiatus & & & $\mathrm{x}$ & & & & & Storeria hidalgoensis* & $\mathrm{x}$ & & & & & & \\
\hline Chiropterotriton chondrostega & & & & & & $\mathrm{x}$ & & Tantilla bocourti & & & & $\mathrm{x}$ & & & \\
\hline Chiropterotriton sp. & $\mathrm{x}$ & & & & & & & Thamnophis cyrtopsis & & & $\mathrm{x}$ & & $\mathrm{x}$ & & \\
\hline Abronia taeniata & & & $\mathrm{x}$ & & & & & Thamnophis eques & & $\mathrm{x}$ & & $\mathrm{x}$ & & & \\
\hline Barisia imbricata & $\mathrm{x}$ & & $\mathrm{x}$ & $\mathrm{x}$ & & & & Thamnophis melanogaster* & $\mathrm{x}$ & & & & & & \\
\hline Gerrhonotus infernalis & $\mathrm{x}$ & & $\mathrm{x}$ & & & & $\mathrm{x}$ & Thamnophis proximus & & & & & $\mathrm{X}$ & & $\mathrm{x}$ \\
\hline Gerrhonotus liocephalus & & & & $\mathrm{x}$ & & & & Nerodia rhombifer & & & & $\mathrm{x}$ & & & $\mathrm{x}$ \\
\hline Phrynosoma orbiculare & $\mathrm{X}$ & $\mathrm{X}$ & $\mathrm{x}$ & $\mathrm{x}$ & & $\mathrm{X}$ & & Thamnophis sumichrasti & & & $\mathrm{x}$ & & & & \\
\hline Sceloporus grammicus & $\mathrm{x}$ & $\mathrm{x}$ & $\mathrm{x}$ & $\mathrm{x}$ & & $\mathrm{x}$ & $\mathrm{x}$ & Trimorphodon tau & $\mathrm{x}$ & & & $\mathrm{x}$ & & & \\
\hline Sceloporus minor & $\mathrm{x}$ & & $\mathrm{x}$ & $\mathrm{x}$ & $\mathrm{x}$ & $\mathrm{x}$ & $\mathrm{x}$ & Tropidodipsas sartorii & $\mathrm{x}$ & & & $\mathrm{x}$ & & & \\
\hline Sceloporus parvus & $\mathrm{x}$ & $\mathrm{x}$ & $\mathrm{x}$ & $\mathrm{x}$ & & $\mathrm{x}$ & $\mathrm{x}$ & Rena dulcis & $\mathrm{x}$ & & & $\mathrm{x}$ & & & \\
\hline Sceloporus scalaris* & $\mathrm{x}$ & & & & & & & Micrurus tener & & & & $\mathrm{x}$ & & & $\mathrm{x}$ \\
\hline Sceloporus spinosus & $\mathrm{x}$ & $\mathrm{x}$ & $\mathrm{x}$ & $\mathrm{x}$ & $\mathrm{x}$ & $\mathrm{x}$ & $\mathrm{x}$ & Crotalus aquilus* & $\mathrm{x}$ & & & & & & $\mathrm{x}$ \\
\hline Sceloporus torquatus & $\mathrm{x}$ & $\mathrm{x}$ & & $\mathrm{x}$ & & & $\mathrm{x}$ & Crotalus atrox & $\mathrm{x}$ & $\mathrm{x}$ & $\mathrm{x}$ & $\mathrm{x}$ & $\mathrm{x}$ & & $\mathrm{x}$ \\
\hline Sceloporus variabilis & & & $\mathrm{x}$ & $\mathrm{x}$ & $\mathrm{x}$ & & $\mathrm{x}$ & Crotalus molossus & & & $\mathrm{x}$ & & & $\mathrm{x}$ & \\
\hline Scincella gemmingeri & & & & & $\mathrm{x}$ & & & Crotalus scutulatus & & & & $\mathrm{x}$ & & & \\
\hline Plestiodon lynxe & $\mathrm{X}$ & & $\mathrm{X}$ & $\mathrm{x}$ & & $\mathrm{X}$ & $\mathrm{x}$ & Kinosternon hirtipes & $\mathrm{x}$ & $\mathrm{x}$ & & $\mathrm{x}$ & $\mathrm{x}$ & & \\
\hline Aspidoscelis gularis & $\mathrm{x}$ & $\mathrm{X}$ & $\mathrm{X}$ & $\mathrm{x}$ & $\mathrm{x}$ & & $\mathrm{x}$ & & & & & & & & \\
\hline Conopsis lineata & $\mathrm{x}$ & & & $\mathrm{x}$ & & $\mathrm{x}$ & & & & & & & & & \\
\hline
\end{tabular}

Fuentes: Los números corresponden a: 1, Fernández (2008), presente estudio; 2, Martín del Campo (1936, 1937); 3, Mendoza (1990) transecto Zacualtipán-Zoquizoquiapan-San Juan Meztitlán; 4, Camarillo (1993) zona xerófila del estado de Hidalgo; 5, Gelover et al. (2000) Meztitlán; 6, Huitzil (2007) región norte de Zimapan; 7, Vite (2008) Reserva de la Biosfera Barranca de Metztitlán.

*= Especies registradas por primera vez para el valle del Mezquital.

por la herpetofauna del valle del Mezquital, "bajo roca" se presentó como el más utilizado por los anfibios. La falta de cuerpos de agua y la escasez de lluvias, así como la marcada selección de este hábitat por ciertas especies limitó su presencia en otros microhábitats.

Los lacertilios ocuparon la mayor cantidad de microhábitats, lo que concuerda con Mendoza (1990). El avistamiento de las serpientes fue poco frecuente debido a sus hábitos esquivos (Camarillo, 1993; Valdespino, 1998).

Los microhábitats más frecuentados fueron "bajo roca", "sobre roca" y "sobre suelo". . Las rocas se utilizan tanto como sitios de asoleo como de refugio, debido a que su exposición directa a los rayos solares, así como la captación y permanencia de calor en la estructura de las rocas permite a cada especie alcanzar la temperatura corporal preferida (Valdespino, 1998). 
En el caso de "sobre suelo", los organismos se encontraron activos, o asoleándose lo cual indica que utilizan algún otro tipo de microhábitat, concordando con lo observado por Mendoza (1990).

La abundancia poblacional de la herpetofauna se ve afectada por la temperatura, la precipitación y la humedad, que son los factores físicos más importantes en la ecología de los anfibios y reptiles (Heatwole, 1976) y que, a su vez, están influidos por la altitud y la topografía. En el presente estudio el factor que limitó mayormente a las poblaciones de anfibios fue la escasez de lluvia durante el año de muestreo (de acuerdo con los datos obtenidos de la estación meteorológica de Ixmiquipan, Hidalgo, para 2004 y 2005), por lo que a excepción de algunas, como Lithobates spectabilis, la mayoría de las especies de anfibios se consideraron raras.

De las 30 especies de reptiles registradas en el estudio, el $50 \%$ se consideraron especies rara, debido a diferentes factores, como la distribución restringida que presentaron en los diferentes tipos de vegetación, el escaso uso de microhábitats y, en el caso particular de las serpientes, sus hábitos esquivos, que dificultan su observación (Vargas, 1998). Otro factor importante, también observado por otros autores, como Vargas (1998) y González (1999), es la intensa persecución del ser humano hacia las serpientes y que también afecta a algunas especies de lagartijas, como Barisia imbricata y Gerrhonotus infernalis.

En la categoría de especies moderadamente abundantes se incluyeron 7 serpientes, las cuales probablemente harán mejor uso de los recursos (Mendoza, 1990), y pese a que la observación no deja de ser complicada, su mayor abundancia aumenta las probabilidades de un avistamiento y asimismo de su supervivencia.

Se listaron 9 especies de reptiles abundantes. Varios autores (Mendoza, 1990; Vargas, 1998; González, 1999), concuerdan en que estas especies frecuentemente se encuentran en 2 o más tipos de vegetación. De este modo, la mayor heterogeneidad espacial, la escasa competencia interespecífica, y la posibilidad de explotar un número mayor de recursos, les permite tener poblaciones abundantes (Lemos y Rodríguez, 1984; Mendoza, 1990).

Las particularidades encontradas en este estudio respecto al tipo de especies que habitan en el valle del Mezquital en relación con otras áreas xerófilas del estado de Hidalgo son una evidencia de la importancia ecológica que presenta esta región, la cual aún esconde una gran riqueza biológica, y no sólo en lo que a anfibios y reptiles se refiere.

\section{Agradecimientos}

Al M. en C. Jesús M. Castillo, por la revisión crítica del manuscrito; al proyecto CONABIO GT002 "Inventario herpetofaunístico del noroeste de Hidalgo" y al proyecto FOMIX-CONACYT Hidalgo 43761 y 95828 "Diversidad biológica del estado de Hidalgo", por el apoyo parcial para las salidas al campo, así como por la beca para Leonardo Fernández.

\section{Literatura citada}

Arroyo, A. 2001. El valle del Mezquital. Una aproximación. Consejo Estatal para la Cultura y las Artes de Hidalgo del Gobierno del Estado de Hidalgo, Pachuca. 143 p.

Camarillo, J. L. 1981. Distribución altitudinal de la herpetofauna comprendida entre Huitzilac, estado de Morelos y La Ladrillera, Estado de México. Tesis, Escuela Nacional de Estudios Profesionales Iztacala, Universidad Nacional Autónoma de México. México, D.F. 44 p.

Camarillo, J. L. 1993. Algunos aspectos biogeográficos de los anfibios y reptiles de la zona xerófila de Hidalgo. In Investigaciones recientes sobre flora y fauna de Hidalgo, México, M. A. Villavicencio, Y. Marmolejo y B. E. Pérez (eds.). Centro de Investigaciones Biológicas, División de Investigación, Universidad Autónoma de Hidalgo, Pachuca. p. 415-432.

Casas, G., G. Valenzuela y A. Ramírez. 1991. Cómo hacer una colección de anfibios y reptiles. Cuadernos del Instituto de Biología 10. Universidad Nacional Autónoma de México, D.F. 68 p.

Colwell, R. y J. Coddington 1994. Estimating terrestrial biodiversity through extrapolation. Philosophical Transactions of the Royal Society of London Series B 345:101-118.

Duellman, E. 1965. A biogeographic account of the herpetofauna of Michoacán, México. University of Kansas Publications of the Museum of Natural History.15:1-82 p.

Duellman, E. 1999. Patterns of distribution of amphibians. A global perspective. Johns Hopkins University Press, Baltimore, Maryland. 633 p.

García, E. 1973. Modificaciones al sistema de clasificación climática de Köppen. Tesis, Universidad Nacional Autónoma de México, México, D.F. 146 p.

Gelover, A., T. Altamirano y M. Soriano. 2000. Uso de los recursos espacio-temporales de la herpetofauna de Metztitlán, Hidalgo. Revista Zoológica 11: 4-11.

González, A. J. 1999. Estudio de los anfibios y reptiles del municipio de Nuevo Urecho, Michoacán. Tesis, Escuela Nacional de Estudios Profesionales Iztacala, Universidad Nacional Autónoma de México, México, D.F. 98 p.

Heatwole, H. 1976, Reptile Ecology. University of Queensland Press. $177 \mathrm{p}$

Huitzil, J. C. 2007. Herpetofauna de doslocalidades en la región norte de Zimapán, Hidalgo. Tesis, Instituto de Ciencias Básicas e ingeniería. Universidad Autónoma del Estado de Hidalgo. México. 90 p.

INEGI(Instituto Nacional de Estadística, Geografíae Informática). 1998. Anuario estadístico del estado de Hidalgo, México, 1990, Aguascalientes, Aguascalientes. p 10-11.

Lemos, J. A y J. L. Rodríguez. 1984. Estudio general de la comunidad herpetofaunística de un bosque templado (mezcla Quercus- Pinus), del Estado de México. Tesis, Escuela 
Nacional de Estudios Profesionales Iztacala, Universidad Nacional Autónoma de México. México, D.F. 41 p.

López, F. 2001. Evaluación y planeación ecológica del uso del suelo, en los municipios de Cardonal, Tasquillo y norte de Ixmiquilpan, Hidalgo. Tesis, Facultad de Ciencias, Universidad Nacional Autónoma de México. México, D.F. $208 \mathrm{p}$.

Martín del Campo, R. 1936. Contribución al conocimiento de la fauna de Actopan, Hidalgo. IV. Vertebrados observados en la época de seca. Anales del Instituto de Biología. 7:271278.

Martín del Campo, R. 1937. Contribución al conocimiento de los batracios y reptiles del valle del Mezquital, Hidalgo. Anales del Instituto de Biología. 8:260-266.

Martínez, R y A. Muñoz. 1998. La herpetofauna de la Reserva el Ocote, Chiapas, México: Una comparación y análisis de su distribución por tipos de vegetación. Boletín de la Sociedad Herpetológica Mexicana. 8: 1-14.

Mendoza, F. 1990. Estudio herpetofaunístico en el transecto Zacualtipán- Zoquizoquipan- San Juan Meztitlán, Hidalgo. Tesis, Escuela Nacional de Estudios Profesionales Iztacala, Universidad Nacional Autónoma de México. México, D.F. $77 \mathrm{p}$.

Muñoz, L. A. 1988. Estudio herpetofaunístico del parque ecológico estatal de Omiltemi. Municipio de Chilpancingo. Guerrero. Tesis, Facultad de Ciencias. Universidad Nacional Autónoma de México, México, D.F. 111 p.
Pineda, E., G. Halffter; C. E. Moreno y F. Escobar. 2005. Transformación del bosque de niebla en agroecosistemas cafetaleros: cambios en las diversidades alfa y beta de tres grupos faunísticos. In Sobre diversidad biológica: el significado de las diversidades alfa, beta y gamma, vol. 4. Monografías Tercer Milenio, G. Halffter, J. Soberón, P. Koleff y A. Melic (eds.). SEA / CONABIO / DIVERSITAS / CONACYT / Zaragoza. 177-190 p.

Rzedowski, J. 1996. Insuficiente el número de Zonas Áridas Protegidas. Ocelotl. 4:25-30.

Shreve, F. 1942. The desert vegetation of North America. Botanical Review 8: 195-246.

Valdespino, C. S. 1998. Anfibios y reptiles de la Sierra del Carmen, Edo. de México. Tesis, Facultad de Ciencias, Universidad Nacional Autónoma de México, México, D.F. 92 p.

Vargas, F. 1998. Estudio herpetofaunístico en el Playon de Mexiquillo y áreas adyacentes en la costa sur del estado de Michoacán, México. Tesis, Facultad de Ciencias, Universidad Nacional Autónoma de México, México, D.F. 111 p.

Vázquez-, Díaz, J. y G. E. Quintero-Díaz. 2005. Anfibios y reptiles de Aguascalientes, Comisión Nacional para el Conocimiento y Uso de la Biodiversidad, México, D.F. 318 p.

Vite, V. D. 2008. Lista anotada de anfibios y reptiles en la reserva de la Biosfera Barranca de Metztitlán, Hidalgo, México. Tesis, Instituto de Ciencias Básicas e Ingeniería. Universidad Autónoma del Estado de Hidalgo. México. 100 p. 